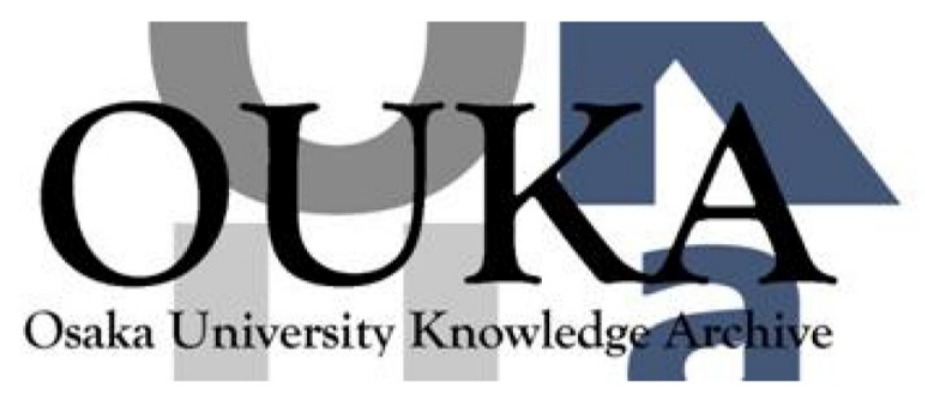

\begin{tabular}{|c|l|}
\hline Title & $\begin{array}{l}\text { High-dose-rate interstitial brachytherapy as a } \\
\text { monotherapy for localized prostate cancer } \\
\text { Treatment description and preliminary results } \\
\text { of a phase I/II clinical trial }\end{array}$ \\
\hline Author(s) & Yoshioka, Yasuo \\
\hline Citation & \\
\hline Issue Date & \\
\hline oaire:version & VoR \\
\hline URL & https://hdl. handle. net/11094/43791 \\
\hline rights & \\
\hline Note & \\
\hline
\end{tabular}

Osaka University Knowledge Archive : OUKA

https://ir. Library. osaka-u. ac. jp/

Osaka University 


\title{
HIGH-DOSE-RATE INTERSTITIAL BRACHYTHERAPY AS A MONOTHERAPY FOR LOCALIZED PROSTATE CANCER: TREATMENT DESCRIPTION AND PRELIMINARY RESULTS OF A PHASE I/II CLINICAL TRIAL
}

\author{
Yasuo Yoshioka, M.D., ${ }^{*}$ Takayuki Nose, M.D., ${ }^{\dagger}$ Ken Yoshida, M.D., ${ }^{*}$ Takehiro Inoue, M.D., ${ }^{*}$ \\ Hideya Yamazaki, M.D., ${ }^{\ddagger}$ Eirchi Tanaka, M.D., $*$ Hiroya Shiomi, M.D., ${ }^{*}$ Atsushi Imai, M.D., $*$ \\ Satoaki Nakamura, M.D., * Shigetoshi Shimamoto, M.D.,* and Toshihiko Inoue, M.D.*
}

*Division of Multidisciplinary Radiotherapy, Biomedical Research Center, and ${ }^{\ddagger}$ Department of Diagnostic Medicine, Osaka University Graduate School of Medicine, Suita, Osaka, Japan; ${ }^{\dagger}$ Department of Radiation Oncology, Osaka Medical Center for Cancer and Cardiovascular Diseases, Osaka, Japan

\begin{abstract}
Purpose: To improve results for localized prostate cancer, a prospective clinical trial of hyperfractionated Iridium-192 high-dose-rate (HDR) brachytherapy as a monotherapy was initiated.

Methods and Materials: Between May 1995 and September 1998, 22 implants were performed on 22 patients with localized prostate cancer (T1:T2:T3:T4 = 4:6:9:3) at Osaka University Hospital. Nineteen patients, who had T3-T4 tumors or pretreatment PSA $\geq 20.0 \mathrm{ng} / \mathrm{mL}$, received hormone therapy. No patient had external beam radiation. Transperineal needle implants using real-time ultrasound guidance were performed, followed by dose optimization program. Patients were irradiated twice a day, with a time interval of more than 6 h. Total dose was $48 \mathrm{~Gy} / 8$ fractions/5 days or $54 \mathrm{~Gy} / 9$ fractions/5 days. Acute toxicity was scored using the Radiation Therapy Oncology Group (RTOG) radiation morbidity scoring criteria. Median follow-up time was 31 months.

Results: HDR brachytherapy as a monotherapy was well-tolerated. No significant intra- or peri-operative complications occurred. No patient experienced acute toxicity of grade 3 or more. PSA levels normalized in $95 \%$ of patients within 20 months after irradiation. Four-year clinical and biochemical relapse-free rates were $95 \%$ and $55 \%$, respectively.

Conclusion: Acute toxicity with this method was acceptable. Further patient accrual and longer follow-up will allow comparison to other techniques. (c) 2000 Elsevier Science Inc.
\end{abstract}

Prostate cancer, Brachytherapy, High-dose-rate, Hyperfractionated, Monotherapy.

\section{INTRODUCTION}

Historically, low-dose-rate (LDR) brachytherapy for early prostate cancer has achieved good clinical local control rates $(1,2)$. It is reasonable to expect the same, or even better, results with ultrasound-guided high-dose-rate (HDR) treatment regimens, having the potential advantages as follows: (a) homogeneous dose distribution using an optimization program, (b) good tumor coverage even in cases with extracapsular invasion or bladder neck invasion, and (c) complete radiation protection for staff and no need for shielding the patient. The safety of HDR brachytherapy is being established in many reports (3-5); however, it is adopted only as a boost in almost every report. If we include external beam irradiation, the dose of brachytherapy has to be reduced to prevent adverse effects, which spoils the advantage of brachytherapy that enables us to deliver an increased dose to the local lesion. It is possible for HDR brachytherapy to cover the locally advanced tumor with enough dose by dwelling sources at the extracapsular position, or even in the bladder pouch; therefore, the supplemental external beam irradiation is not essential. In this philosophy, we planned HDR brachytherapy as a monotherapy to the localized prostate cancer without external beam irradiation.

\section{METHODS AND MATERIALS}

\section{Patient characteristics and selection}

Between January 1994 and September 1998, 28 patients with prostate cancer were treated with interstitial brachytherapy. Twenty-two of 28 patients met strict protocol-entry criteria and are the subject of this report.

Protocol eligibility included the following: (a) having clinical TNM stage T1-T3 and a part of T4 tumor (bladder
Reprint requests to: Yasuo Yoshioka, M.D., Division of Multidisciplinary Radiotherapy, Biomedical Research Center, Osaka University Graduate School of Medicine (D10), 2-2 Yamada-oka Suita, Osaka 565-0871, Japan. Tel: +81-6-6879-3482; Fax: +816-6879-3489; E-mail: yoshioka@radonc.med.osaka-u.ac.jp
Accepted for publication 8 May 2000. 
neck invasion) without nodal metastasis or other distant metastasis by clinical, biochemical, and imaging studies, including all MRIs, CTs, and bone scans in all patients; (b) patients had to be candidates for epidural anesthesia; (c) a pretreatment transrectal ultrasound and serum prostate-specific antigen (PSA) level were required in all patients; and (d) informed consent was mandatory. Patients were considered nonprotocol for various reasons, including: (a) prior pelvic radiotherapy for another malignancy, (b) prior surgery/TUR-P (transurethral resection of prostate) to prostate gland, and (c) prostate cancer recurrence. Nonprotocol patients will not be considered further in this report.

The median age at diagnosis was 69 years (range 45-81). All patients had biopsy-proven adenocarcinoma of the prostate. Four patients had well-differentiated adenocarcinoma, 10 patients had moderately-differentiated adenocarcinoma, and 8 patients had poorly-differentiated adenocarcinoma. Using UICC TNM classification of 1997, 4 patients were classified as T1, 6 patients as T2, 9 patients as T3, and 3 patients as T4. The pretreatment PSA level ranged from 7.0 to $150.0 \mathrm{ng} / \mathrm{mL}$ (median $30.9 \mathrm{ng} / \mathrm{mL}$ ), including 2 cases with $4.0-9.9 \mathrm{ng} / \mathrm{mL}, 6$ cases with $10.0-19.9 \mathrm{ng} / \mathrm{mL}$, and 14 cases with $20.0 \mathrm{ng} / \mathrm{mL}$ or more.

\section{Hormonal therapy}

The patients whose T-stages had been T1-T2 and pretreatment PSA levels $<20.0 \mathrm{ng} / \mathrm{mL}$ were treated with radiotherapy alone (3 patients). The others (patients whose T-stages had been T3-T4 or whose pretreatment PSA levels $\geq 20.0 \mathrm{ng} / \mathrm{mL}$ ) received neoadjuvant and adjuvant hormonal therapy (19 patients) (6). Neoadjuvant hormonal therapy was based on maximum androgen blockade including LH-RH agonist (leuprorelin acetate or goserelin acetate) and antiandrogen (chlormadinone acetate or flutamide). Leuprorelin acetate was given at a dosage of $3.75 \mathrm{mg}$ s.c. every 4 weeks, and $125 \mathrm{mg}$ flutamide was given orally three times daily. If ineffectiveness or adverse effects were observed, leuprorelin acetate was changed to $3.6 \mathrm{mg}$ goserelin acetate and/or flutamide was changed to $50 \mathrm{mg}$ chlormadinone acetate orally twice daily. In the 5 days of brachytherapy treatment, all hormonal therapy was interrupted. After brachytherapy was completed, LH-RH agonist was restarted, while antiandrogen was discontinued. The subgroup whose T-stage had been T1-T2 and whose PSA levels reached less than $0.7 \mathrm{ng} / \mathrm{mL}$ (which was the minimal detectable value in our institution) discontinued all hormonal therapy unless they hoped to continue it. As for the other patients, only LH-RH analog was administered, unless the patient refused it. If the biochemical failure was confirmed, antiandrogen was restarted with or without starting other hormonal therapy or chemotherapy.

\section{Treatment technique}

Brachytherapy was performed under epidural anesthesia. At the time of operation, the patient was placed in a dorsal lithotomy position, with the hips flexed as much as possible, and the perineal region was prepared and draped in the usual sterile fashion. A balloon catheter was inserted into the bladder with iodinated contrast in the balloon. The bladder was distended with 100-cc sterile saline. A stepper carriage with an acrylic rectangular template attached was mounted to the operating room table via an arm with locking universal joints. The template (TAISEIMEDICAL, Osaka, Japan) has 88 needle holes spaced at 5-mm intervals without disturbing the passage of the ultrasound probe. The ultrasound probe (mechanical radial scanner, ASU-64B ${ }^{\circledR}$, ALOCA, Tokyo, Japan) was connected to the template/stepper carriage and the intrarectal portion was covered with a standard condom. The probe was inserted into the rectum and maneuvered in such a way as to place the template in contact with the perineum. The probe was manipulated to obtain the best possible ultrasound image, without undue compression of the posterior prostate. The fixation device was locked and only longitudinal (superior-inferior) movement was permitted thereafter. A series of transverse views of these levels was measured and stored. The depth of probe insertion was marked for distal end of the seminal vesicle, the base, the apex, and the largest cross-section of the prostate gland (called the midgland). Prior to needle placement, the probe was placed at the midgland and the contours of the prostate and seminal vesicle were outlined. The target volume of the implantation was the prostate gland with or without the medial aspects of the seminal vesicle. The medial aspect was defined as a medial half of the seminal vesicle, which was visualized on transrectal ultrasound (TRUS) in the dorsal direction of the prostate gland and the bladder neck up to 20 or $30 \mathrm{~mm}$ cranial from the base of the prostate gland. The target volume was the prostate alone in T1 cases and in the majority of $\mathrm{T} 2$ cases, while in $\mathrm{T} 3$ or T4 cases, and in a part of T2 cases with suspected seminal vesicle microinvasion, the target volume involved the medial aspect of the seminal vesicle other than the prostate, including the extracapsularly invaded area. To ensure adequate implantation of the medial aspects of the seminal vesicles, these were imaged by ultrasound, and needle placement was documented (Fig. 1). Metallic markers were implanted in the apex, the distal end of the seminal vesicle, and critical point for each patient to visualize on a roentgenogram. The metallic needles (Trocar Point Needles ${ }^{\circledR}$, Nucletron, The Netherlands) were inserted to $10 \mathrm{~mm}$ cephalad to the prostate and seminal vesicles. As each needle was placed, it was identified by its template coordinates (3).

After all of the needles were placed under ultrasound guidance, the patient was taken to the simulation room, where roentgenograms were taken with dummy sources packed in the needles. A CT scan was then performed to ensure the relationship between needles and the prostate, seminal vesicles, bladder, rectum, and bowels. The radiation oncologists determined areas of source dwell on the roentgenograms and the $\mathrm{CT}$. Physicists reconstructed the actual location of each needle by recognizing the dummy sources on the roentgenograms, and optimized dwell weights (Fig. 1). The rectal and bladder dose was also calculated. 

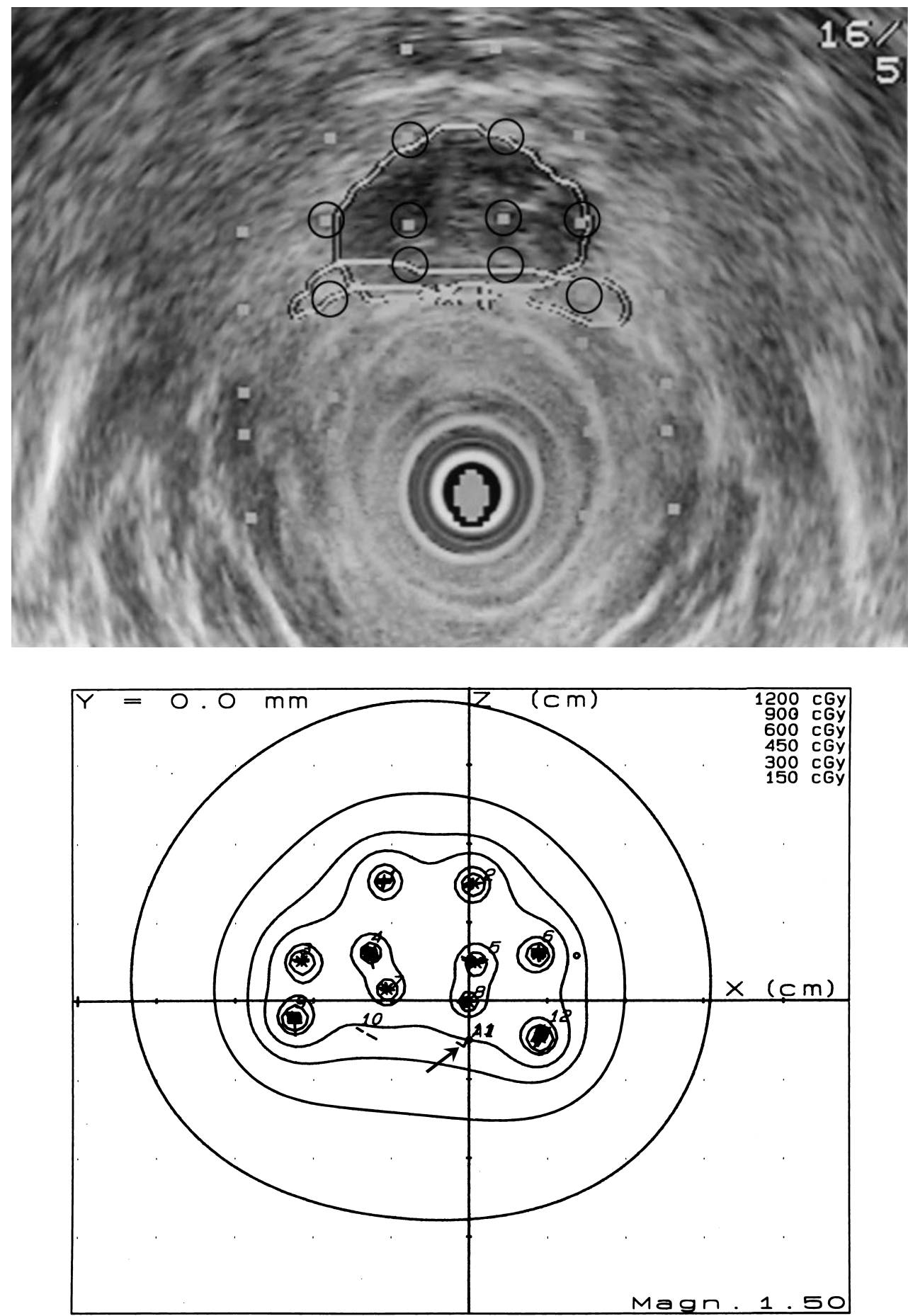

Fig. 1. (a) TRUS showing the prostate enclosed by a superimposed line at the largest cross-section of the prostate and the seminal vesicle. The superimposed square dots represent some of the grids in the template. The open circles indicate the places where the needles were actually implanted in this case. (The actual intervals of needles were $5 \mathrm{or} 10 \mathrm{~mm}$.) (b) The isodose plots of the same plane with (a). The arrow indicates the prescribed dose point and the curve crossing it indicates the prescribed dose line.

Patients were restricted to bed for 5 days, from Monday to Friday, and irradiated twice a day, with an interval of at least $6 \mathrm{~h}$ between irradiation (Fig. 2). The treatment consisted of (a) 8 fractions of 6.0 Gy each, 48 Gy in total, in the first 7 cases, and (b) 9 fractions of 6.0 Gy each, 54 Gy in total, to the prostate in the latter 15 cases. Epidural anesthesia was continued to the last day of irradiation (for 5 days). Prophylactic antibiotic was administered twice daily from the day of implant through day 7. To suppress defecation, the patients were given low-residuum meals from 4 days before implant to the last day of irradiation and morphine chloride $20-40 \mathrm{mg}$ orally three 
(Mon)

(Tue)

(Wed)

(Thu)

(Fri)

AM implantation (2)

$6 \mathrm{~Gy}$

(4)

$6 \mathrm{~Gy}$

(6)

$6 \mathrm{~Gy}$

(8)

PM

planning

(1)

$6 \mathrm{~Gy}$
$6 \mathrm{~Gy}$
(5)

6 Gy
6 Gy

\section{(9) \\ 6 Gy \\ detachment}

Needle implanted

\section{Continuous epidural anesthesia}

Fig. 2. Treatment schedule. The patients received a total dose of 54 Gy/9 fractions/5 days (from Monday to Friday) under continuous epidural anesthesia. The time interval between the irradiation is at least $6 \mathrm{~h}$.

times daily from the day of implant to the last day of irradiation.

\section{Follow-up and toxicity analysis}

Follow-up was performed by the radiation oncologist and urologist at least once a month. Digital rectal examination was performed every time. PSA level was examined monthly and MRI every 6 months during follow-up in all patients. Biochemical failure was defined as three times successive elevation of PSA levels from nadir of PSA after treatment, and the date of failure was defined as the midpoint between the postirradiation nadir PSA and the first of three consecutive rises (7). PSA was examined by AIAPACK PA (TOSOH, Japan), and the normal range was defined as $2.1 \mathrm{ng} / \mathrm{mL}$ or less in this report.

Acute and chronic toxicities were scored using Radiation Therapy Oncology Group (RTOG) guidelines (8). Acute toxicity was defined as those symptoms that presented during or after treatment that completely resolved by 6 months post-treatment. Acute toxicity was graded according to the most severe reaction observed. Treatment-related toxicities that persisted or presented beyond 6 months following treatment completion were considered chronic.

\section{Statistical methods}

Clinical local control and biochemical control rates were calculated by the method of Kaplan and Meier (9).

\section{RESULTS}

\section{Feasibility}

All patients who started this treatment completed all courses of the regimen. Although some patients complained of lumbago due to continuous lying in bed during the course of treatment, it was controllable and did not interrupt the treatment regimen. Three patients felt numbness at the right or left leg due to the continuous epidural anesthesia, which was resolved easily by decreasing the dose of anesthetic, and did not interrupt the treatment regimen. In some cases, defecation in the treatment course was experienced, which did not disturb the treatment.

Acute toxicity analysis

The median follow-up time was 31 months (range 1958). No significant intraoperative or perioperative complications that required treatment modification occurred. Toxicity of grade 3 or more was not observed in any patient. Grade 2 acute toxicities were experienced in $8(36 \%)$ patients a few days after treatment; all of them healed without special treatment (Table 1). The number of patients with grade 1 acute toxicity-alone was $3(14 \%)$. All of the patients who had gastrointestinal toxicities (4 patients) also had grade 1 genitourinary toxicities. The total number of patients that showed any grade of acute toxicities was 11 $(50 \%)$. Table 2 presents in detail the acute toxicity encountered with this treatment approach. We did not consider the temporary event of hematuria, during or just after implantation or detachment of the needles, which was caused by mechanical stimulation of the needles and recovered without treatment in a few hours, as acute radiation toxicity.

\section{Chronic toxicity}

Grade 2 rectal ulcer was experienced in one case 22 months after brachytherapy, and occasional grade 1 rectal

Table 1. Acute toxicity results $(n=22)$

\begin{tabular}{lccc}
\hline & Gastrointestinal & Genitourinary & Overall \\
\hline Grade 5 & 0 & 0 & 0 \\
Grade 4 & 0 & 0 & 0 \\
Grade 3 & 0 & 0 & 0 \\
Grade 2 & $3(14 \%)$ & $5(23 \%)$ & $8(36 \%)$ \\
Grade 1 & $1(5 \%)$ & $6(27 \%)$ & $6(27 \%)$ \\
Total & $4(18 \%)$ & $11(50 \%)$ & $11(50 \%)$ \\
\hline
\end{tabular}

Grade: RTOG acute radiation morbidity scoring criteria. 
Table 2. Contents of acute toxicities

\begin{tabular}{lll} 
Grade 3 or more & None & \\
Grade 2 & $2 *$ & Dysuria \\
& 2 & Rectal pain \\
& 1 & Perineal pain \\
& 1 & Increased urinary frequency/urgency \\
& 1 & Gross hematuria $\dagger$ \\
Grade 1 & 1 & Urinary tract infection \\
& 5 & Increased urinary frequency/urgency \\
& 3 & Urinary retension \\
& 1 & Dysuria \\
& 1 & Rectal pain \\
& 1 & Hematuria \\
\hline
\end{tabular}

* The total number exceeds that of Table 1 because some patients revealed multiple events.

${ }^{\dagger}$ Temporary gross hematuria needed medication.

* Temporary hematuria without need for medication.

bleeding has been reported in another case since 24 months after brachytherapy. Impotence was estimated in only 5 patients, because all other patients had lost potency before brachytherapy due to aging or could not be estimated due to continuing adjuvant hormonal therapy. Two patients, who had not received hormonal therapy, have kept potency after brachytherapy thus far (24 and 55 months post-brachytherapy, respectively). Another two patients, who had received neoadjuvant and adjuvant hormonal therapy and then discontinued adjuvant hormonal therapy, recovered potency (21 and 36 months post-brachytherapy, respectively). One patient lost potency after brachytherapy; however, the time when he lost potency had been masked by 5 months of neoadjuvant hormonal therapy and 1 month of adjuvant hormonal therapy. No other chronic toxicity, such as incontinence or urethral narrowing has been observed thus far.

\section{Rectal and urethral dose calculations}

The dose irradiated for urethra distributed between 54 and 81 Gy (for the group irradiated with a total dose of 54 Gy).

The dose irradiated for anterior surface of rectal mucosa was under the prescribed dose (54 Gy) in all cases.

\section{Irradiated volume}

The median number of needles placed was 16 (range $8-18)$. Median V100 (100\% isodose volume) was $55.7 \mathrm{~cm}^{3}$ (range $28-82 \mathrm{~cm}^{3}$ ), and median V200 (200\% isodose volume) was $5.0 \mathrm{~cm}^{3}$ (range $2.3-7.4 \mathrm{~cm}^{3}$ ). All cases that had more than $60 \mathrm{~cm}^{3}$ irradiated volume resulted in grade 1 or 2 complications (Fig. 3).

\section{Clinical local control}

All 22 patients were controlled locally; that is, no evidence of tumor was detected either on digital-rectal examination or on MRI. One case developed multiple bone metastasis 7 months after the treatment but showed no evidence of local recurrence.

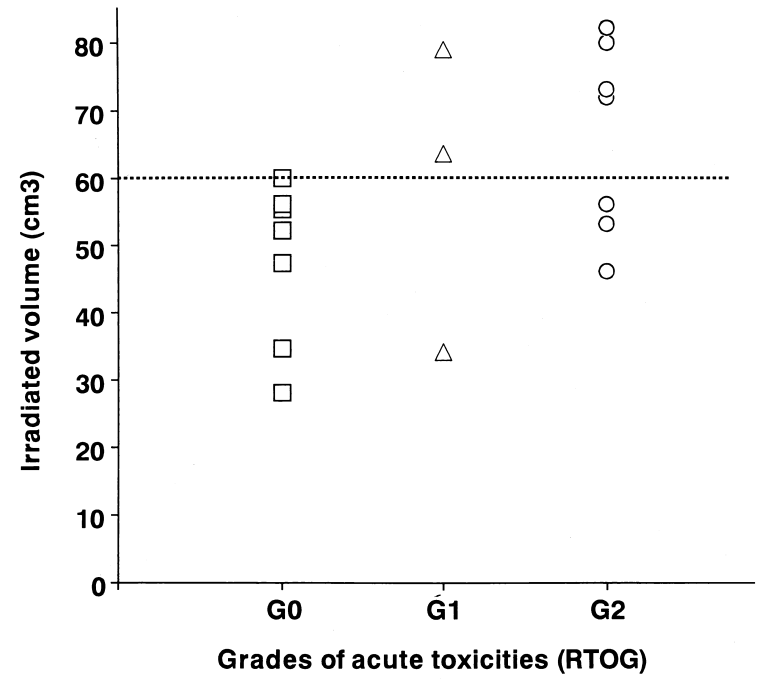

Fig. 3. Relationship between acute complications and irradiated volumes. All cases that had more than $60 \mathrm{~cm}^{3}$ irradiated volumes resulted in grade 1 or 2 complications.

\section{Biochemical control}

Biochemical control rate was shown in Fig. 4, excluding one patient who developed multiple bone metastasis. Two-, three-, and four-year control rates were $65 \%, 55 \%$, and $55 \%$, respectively.

In 22 patients, 19 patients had been treated with hormone therapy before HDR brachytherapy. In this group, the median pretreatment PSA level had been $48.2 \mathrm{ng} / \mathrm{mL}$ (range 7.2-150.0), and the median span of the neoadjuvant hormonal therapy was 7 months (range 1-19). PSA levels had been normalized before brachytherapy in 12 of 19 cases. In the remaining 7 cases, PSA levels had not been normalized by neoadjuvant hormone therapy but normalized by brachytherapy in 6 cases, while one case did not reach the normal range. The time to PSA nadir after HDR brachytherapy ranged from 1 to 20 months (median 9). Four patients stopped adjuvant hormonal therapy after the median span of 14 months of adjuvant hormonal therapy (range 1-32), and the other 15 patients continued adjuvant hormonal therapy.

Three patients were treated with HDR brachytherapyalone without hormone, and their PSA levels were all normalized after HDR brachytherapy. Their pretreatment PSA levels were 7.0, 18.6, and $19.3 \mathrm{ng} / \mathrm{mL}$, respectively, and the time to PSA nadir after HDR brachytherapy was 6,15 , and 14 months, respectively.

\section{DISCUSSION}

To improve the local control rate after radiotherapy, dose escalation is a reasonable approach because dose response relationship for radiotherapy exists $(10,11)$. However, an increased external beam irradiation dose will at the same time increase the toxicity rate $(10,11)$, and the organ motion 


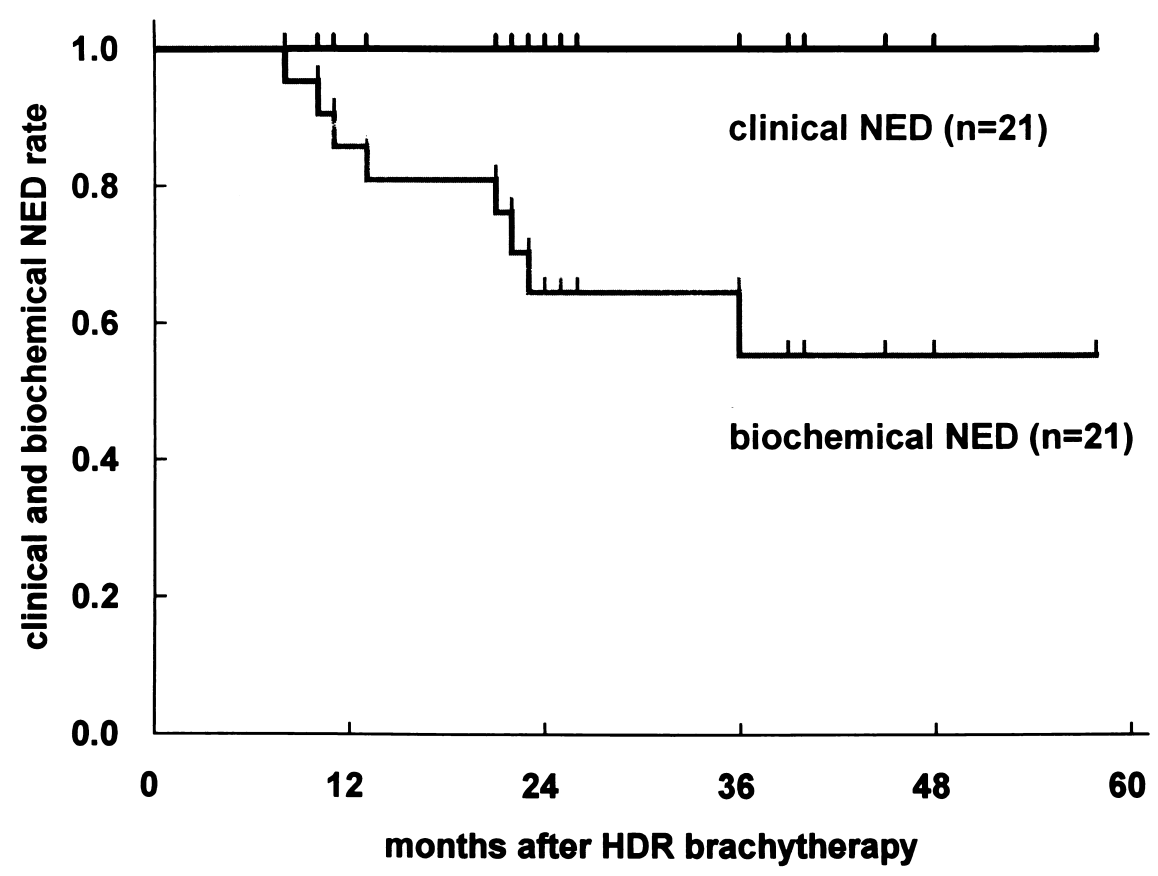

Fig. 4. Kaplan-Meier plot of clinical NED (no evidence of disease) rate and biochemical NED rate. The one patient who developed multiple bone metastasis without local recurrent signs was excluded. Two-, three-, and four-year biochemical NED rates were $65 \%, 55 \%$, and $55 \%$, respectively.

makes it difficult to shrink the field size for reducing adverse effects (12).

Interstitial brachytherapy has the potential to solve this problem; that is, it enables us to administer higher dose in a small volume (the prostate), and to spare the adjacent structures due to rapid dose fall-off outside the target volume.

Past experience using LDR prostate implantation has resulted in good pathologic and clinical local control rates $(1,2)$, but not satisfactory ones in the locally advanced or high-risk cases, which may be attributed to inhomogeneous dose distribution and insufficient tumor covering. It is reasonable to expect the same, or even better, results with ultrasound-guided HDR treatment regimens, because it has achieved homogeneous dose distribution by an optimization program, the rigidity of metallic needles to grasp the target leads to the preservation of the calculated dose distribution throughout the treatment period. It is difficult to estimate the actual irradiated dose in LDR brachytherapy because of post-implant edema of the prostate, which may have a significant effect on the post-implant dosimetry (13). It is also difficult to estimate the actual irradiated dose in external beam radiotherapy because of the daily variability of the position of the prostate (12). The HDR needle implantation technique also has advantages over seed implantation in the area of tumor coverage, because the treatment source can be delivered deep enough to cover the margin of the target, moving freely into the needles even in the extracapsular tissue or in the bladder pouch.

On the other hand, according to the current radiobiological theory, HDR has a disadvantage of a lower therapeutic ratio, and the short overall treatment time of 5 days would disadvantageously affect treatment of the prostate cancer. However, the advantages of HDR brachytherapy in radiation physics mentioned above possibly compensate for such radiobiological disadvantages.

In the treatment of T3-T4 prostate cancer, in which radical prostatectomy or LDR brachytherapy may be a contraindication because of the incapability to treat extracapsularly invasive tumor, the combination of HDR brachytherapy and hormonal therapy may be considered as one of the possible radical therapies. Supplemental hormonal therapy may enhance the effect of radiotherapy without limiting it, while the supplemental external beam radiotherapy would limit the total dose of brachytherapy (6).

The results presented in this report indicate acceptable short-term tolerance to the radiation doses delivered by our technique using hyperfractionated HDR Iridium-192 brachytherapy as a monotherapy. The treatment-related toxicities were primarily gastrointestinal and genitourinary in nature and were, as expected, less than a course of whole pelvis external irradiation. It was an encouraging result that no patient showed acute toxicity of grade 3 or more. However, the irradiated volume of more than $60 \mathrm{~cm}^{3}$ may highly predict grade 1 or 2 acute toxicity in our method. As for chronic toxicities, the low rate of rectal bleeding or impotence, and the fact that no patient has revealed incontinence or urethral narrowing is expected, although the follow-up time is too short for estimation.

The local tumor response in this series as assessed by serial PSA measurements after irradiation and clinical examinations appears encouraging, although further patient accrual and longer follow-up are necessary to confirm these 
results. The rate of PSA normalization (95\%) in our results is highly encouraging, considering that our patients include many T3-T4 or high pretreatment PSA level ( $\geq 20 \mathrm{ng} / \mathrm{mL}$ ) cases $(82 \%)$, which may unfavorably affect PSA normalization rates. However, it must be taken into consideration that we used hormone as a neoadjuvant for the patients whose pretreatment PSA levels were $20.0 \mathrm{ng} / \mathrm{mL}$ or more until PSA levels were reduced to less than $20.0 \mathrm{ng} / \mathrm{mL}$. And we also used hormone for all T3-T4 patients. The 6 cases whose PSA levels had not been normalized by hormone but were normalized by brachytherapy, and the 3 cases whose PSA levels were normalized by brachytherapy alone, suggest the early efficacy of this treatment regimen even without adding hormone. The present report also adds to the paucity of information regarding PSA normalization fol- lowing irradiation treatment approaches that use HDR brachytherapy as a monotherapy.

\section{CONCLUSION}

Acute toxicity in 22 patients treated with this unique approach, using HDR brachytherapy without external beam irradiation, has been acceptable within the established protocol guidelines. The initial tumor response, as assessed by PSA measurement, appears promising and further patient accrual and follow-up are necessary to determine local control, biochemical control, survival, and chronic toxicity rates with this treatment regimen. If acceptable local control and toxicity rates are observed, we plan to conduct a Phase III randomized study to compare this treatment approach with conformal external beam irradiation for locally advanced prostate cancer.

\section{REFERENCES}

1. Blasko J, Ragde H, Luse R, et al. Should brachytherapy be considered a therapeutic option in localized prostate cancer. Urol Clin NA 1996;23:633-650.

2. Bayer DC, Priestley JB. Biochemical disease-free survival following I-125 prostate implantation. Int J Radiat Oncol Biol Phys 1997;37:559-563.

3. Stromberg J, Martinez A, Gonzalez J, et al. Ultrasound-guided high dose rate conformal brachytherapy boost in prostate cancer: Treatment description and preliminary results of a phase I/II clinical trial. Int J Radiat Oncol Biol Phys 1995;33: 161-171.

4. Kovacs G, Wirth B, Bertermann H, et al. Prostate preservation by combined external beam and HDR brachytherapy at nodal negative prostate cancer patients-an intermediate analysis after ten years experience. (Abstr.) Int J Radiat Oncol Biol Phys 1996;36(Suppl. 1):198.

5. Mate TP, Gottesman JE, Hatton J, et al. High dose-rate afterloading Ir-192 prostate brachytherapy: Feasibility report. Int J Radiat Oncol Biol Phys 1998;41:525-533.

6. Laverdiere J, Gomez JL, Cusan L, et al. Beneficial effect of combination hormonal therapy administered prior and following external beam radiation therapy in localized prostate cancer. Int J Radiat Oncol Biol Phys 1997;37:247-252.

7. American Society for Therapeutic Radiology and Oncology
Concensus Panel. Consensus statement: Guidelines for PSA following radiation therapy. Int J Radiat Oncol Biol Phys 1997;37:1035-1041.

8. Cox JD, Stetz J, Pajak TF. Toxicity criteria of the radiation therapy oncology group (RTOG) and the European organization for research and treatment of cancer (EORTG). Int $J$ Radiat Oncol Biol Phys 1995;31:1341-1346.

9. Kaplan EL, Meier P. Nonparametric estimation from incomplete observation. J Am Stat Assoc 1958;53:457-481.

10. Pollack A, Zagars GK. External beam radiotherapy dose response of prostate cancer. Int J Radiat Oncol Biol Phys 1997;39:1011-1018.

11. Hanks GE, Hanlon AL, Schultheiss TE, et al. Dose escalation with 3D conformal treatment: Five year outcomes, treatment optimization, and future directions. Int J Radiat Oncol Biol Phys 1998;41:501-510.

12. Dawson LA, Mah K, Franssen E, et al. Target position variability throughout prostate radiotherapy. Int J Radiat Oncol Biol Phys 1998;42:1155-1161.

13. Waterman FM, Yue N, Corn BW, et al. Edema associated with I-125 or Pd-103 prostate brachytherapy and its impact on post-implant dosimetry: An analysis based on serial CT acquisition. Int J Radiat Oncol Biol Phys 1998;41:1069-1077. 